\title{
Phosphorus magnetic resonance spectroscopy in the investigation of temporal lobe epilepsy: 'reading between the lines' of metabolic abnormalities
}

\author{
Espectroscopia de fósforo por ressonância magnética na investigação de epilepsia do \\ lobo temporal: 'lendo nas entrelinhas' das alterações metabólicas
}

Luciana Ramalho Pimentel-Silva; Fernando Cendes

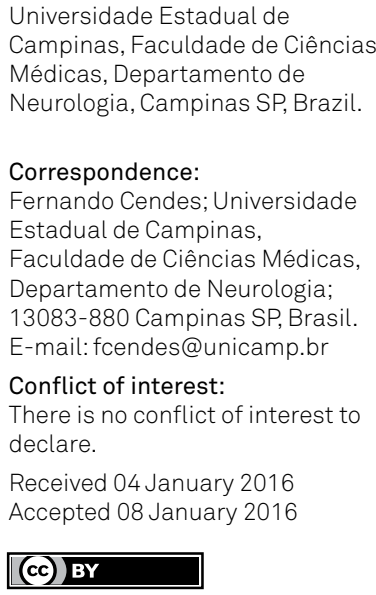

7 he natural course of mesial temporal lobe epilepsy (MTLE) is usually associated with a precipitating injury in childhood, which later develops to become the most common form of focal epilepsy in adulthood ${ }^{1}$. Hippocampal sclerosis (HS) is the main underlying lesion detected in both histopathology and magnetic resonance imaging (MRI) evaluation of these patients. A significant proportion of patients MTLE become refractory to antiepileptic drugs (AEDs). Surgery is the most effective treatment in this scenario, with the majority of patients becoming seizure free postoperatively. However, there are many open questions about the neurobiology of MTLE. Focusing on brain imaging, for example, most MTLE patients present with HS although in some the MRI is negative ${ }^{2}$. Moreover, not only the hippocampus seems to be involved in the physiopathology of TLE but also extratemporal regions ${ }^{2,3}$. Besides, for surgical treatment to succeed is mandatory to correctly lateralize seizure focus, which is more complex in MRI negative patients. Magnetic resonance spectroscopy (MRS) ist an important tool in the investigation of these broader and subtle changes that are missed by structural MRI techniques. Exploiting the magnetic properties of several nuclei, MRS is a useful tool when it comes to provide metabolic information in vivo without invasive intervention. The most common is proton magnetic resonance spectroscopy (1H-MRS), due to ${ }^{1} \mathrm{H}$ natural abundance in biological tissues ${ }^{2}$. Phosphorus MRS ( $\left.{ }^{31} \mathrm{P}-\mathrm{MRS}\right)$ is not so commonly employed in brain tissue evaluation; nevertheless, it is able to add important information about energy metabolism. The metabolites visible in a high resolution spectrum from ${ }^{31} \mathrm{P}-\mathrm{MRS}$ are basically phosphorus compounds: total adenosine triphosphate (total ATP, composed by $\gamma-+\alpha-+\beta$-ATP, according to the position of the three phosphate groups), inorganic phosphate (Pi), PCr (phosphocreatine), phosphodiesters (PDE, the sum of glycerophosphocholine plus glycerophosphoethanolamine) and phosphomonoesters (PME, composed by phosphocholine plus phosphoethanolamine $)^{4}$. These metabolites are mainly involved in mitochondrial processes and cellular membrane turnover. Fewer studies addressed the issue of metabolic changes in epilepsy using ${ }^{31} \mathrm{P}-\mathrm{MRS}$ than those using ${ }^{1} \mathrm{H}-\mathrm{MRS}$. Data from ${ }^{1} \mathrm{H}$-MRS show a decrease in $\mathrm{N}$-acetyl aspartate (NAA) referred as a result of neuronal loss and/or neuronal mitochondrial dysfunction, in other words, energy metabolism disturbance. NAA decrease is a key finding in several types of epilepsy, often associated with the lateralization of seizure focus and AED response ${ }^{2}$. Since mitochondria are the energetic engine of the cell, further evaluation of brain energy metabolism may help to shed lights on seizures underlying mechanisms. In spite of having less spatial resolution than ${ }^{1} \mathrm{H}-\mathrm{MRS}$, studies on ${ }^{31} \mathrm{P}-\mathrm{MRS}$ show that it is able to lateralize the seizure focus and also to characterize the role of brain energy metabolism alterations in $\mathrm{MTLE}^{5,6}$. In this issue of Arquivos de Neuro-Psiquiatria, Park et al. ${ }^{7}$ showed abnormal metabolism as measured by ${ }^{31} \mathrm{P}-\mathrm{MRS}$ in extratemporal areas in patients with MTLE. Evidences from MRS and other imaging modalities give support for the more widespread functional impairment extending beyond the mesial temporal structures in patients with MTLE ${ }^{6,8}$. More detailed high-resolution quantitative MRI analyses reveals a 
network of gray matter atrophy and dysfunction that involves mesial temporal and other structures interconnected with the limbic system, including thalamus and striatum ${ }^{9}$; a finding that was expanded further by the study of Park et al. ${ }^{7}$. The authors studied a significant number $(n=33)$ of well-defined patients with refractory seizures and MRI signs of HS and compared to 31 healthy controls. They performed multivoxel ${ }^{31} \mathrm{P}-\mathrm{MRS}$ in the anterior insula-basal ganglia (AIBG), posterior insula-basal ganglia (PIBG) and frontal lobes (FL), finding differences in Pi, the ratio $\mathrm{PCr} / \gamma$-ATP, total ATP and $\gamma$-ATP in the patients group when compared to controls. The metabolites changes were found in all regions studied although less pronounced in FL.
The authors hypothesized that Pi decrease could be a compensatory mechanism observed in the interictal period as a consequence of the high energy demand during the ictal phase. In fact, these data support the underlying basis of the metabolic disturbance found in ${ }^{1} \mathrm{H}-\mathrm{MRS}$ and FDG-PET studies ${ }^{8,9,10}$. The study of Park et al. ${ }^{7}$ lacks a clinical correlation with ${ }^{31} \mathrm{P}-\mathrm{MRS}$ findings, as the authors recognize. The work of Park et al. ${ }^{7}$ rekindles the use of brain ${ }^{31} \mathrm{P}-\mathrm{MRS}$ for the study of dysfunctions in brain metabolism related to epilepsy, and in particular to MTLE. Further studies are necessary to elucidate better the relationships of the ${ }^{31} \mathrm{P}-\mathrm{MRS}$ abnormalities with seizures, use of AEDs, structural damage and other clinical variables.

\section{References}

1. Andrade-Valença LPA, Valença MM, Velasco TR, Leite JP. [Mesial temporal lobe epilepsy with hippocampal sclerosis]. J Epilepsy Clin Neurophysiol 2006;12(1):31-6. Portuguese. doi:10.1590/S1676-26492006000100007

2. Pimentel-Silva LR, Cendes F. Proton magnetic resonance in epilepsy: key findings. J Epilepsy Clin Neurophysiol. 2015;21(4):136-43.

3. Mueller SG, Laxer KD, Cashdollar N, Flenniken DL, Matson GB, Weiner MW. Identification of abnormal neuronal metabolism outside the seizure focus in temporal lobe epilepsy. Epilepsia. 2004;45(4):355-66. doi:10.1111/j.0013-9580.2004.27603.x

4. Ren J, Sherry AD, Malloy CR. (31) P-MRS of healthy human brain: ATP synthesis, metabolite concentrations, $\mathrm{pH}$, and $\mathrm{T} 1$ relaxation times. NMR Biomed. 2015;28(11):1455-62. doi:10.1002/nbm.3384

5. Chu WJ, Hetherington HP, Kuzniecky RI, Simor T, Mason GF, Elgavish GA. Lateralization of human temporal lobe epilepsy by 31P NMR spectroscopic imaging at 4.1 T. Neurology. 1998;51(2):472-9. doi:10.1212/WNL.51.2.472
6. Pan JW, Kim JH, Cohen-Gadol A, Pan C, Spencer DD, Hetherington HP. Regional energetic dysfunction in hippocampal epilepsy. Acta Neurol Scand. 2005; 111(4):218-24. doi:10.1111/j.1600-0404.2005.00398.x

7. Park EJ, Otaduy MCG, Lyra KP, Andrade CS, Castro LH, Passareli $\checkmark$ et al. Extratemporal abnormalities in phosphorus magnetic resonance spectroscopy of patients with mesial temporal sclerosis. Arq Neuropsiquiatria. 2015;74(2):93-8. doi:10.1590/0004-282X20150201

8. Cendes F, Knowlton RC, Novotny E, Li LM, Antel S, Sawrie S et al. Magnetic resonance spectroscopy in epilepsy: clinical issues. Epilepsia. 2002;43(Suppl 1):32-9. doi:10.1046/j.1528-1157.2002.043s1032.x

9. Cendes F, Sakamoto AC, Spreafico R, Bingaman W, Becker AJ. Epilepsies associated with hippocampal sclerosis. Acta Neuropathol. 2014;128(1):21-37. doi:10.1007/s00401-014-1292-0

10. Henry TR, Roman DD. Presurgical epilepsy localization with interictal cerebral dysfunction. Epilepsy Behav. 2011;20(2):194-208. doi:10.1016/j.yebeh.2010.12.008 